\title{
Reflexiones nerviosas De huecos y de rellenos.
}

\author{
Juan Medrano \\ Médico psiquiatra. Red de Salud Mental de Bizkaia. \\ oban@telefonica.net
}

Neil deGrasse Tyson es un astrofísico, escritor y divulgador científico estadounidense que en la actualidad dirige el Planetario Hayden en el Centro Rose para la Tierra y el Espacio, en Nueva York. Es especialmente conocido por sus intervenciones en medios de comunicación, en las que con un estilo ameno y desenfadado se afana por extender información científica sobre temas no siempre fáciles de digerir. Viene a ser, para que nos entendamos, un a modo de Carl Sagan contemporáneo, pero en afroamericano, zumbón y con bigote. En sus charlas Tyson habla a menudo de lo que llama el "Dios de los huecos" (God of gaps), para referirse al recurso histórico a Dios para rellenar la falta de conocimiento científico del ser humano; lo cuenta, por ejemplo, en: https://www. youtube.com/watch? $\mathrm{v}=$ pwCt7WWiPXA. Según su razonamiento, a lo largo de la Historia, el desconocimiento sobre el mecanismo de funcionamiento de los fenómenos naturales ha hecho necesario recurrir a la figura de Dios (o de los dioses) para explicar cualquier evento cuyo sentido no fuera asequible al conocimiento humano. Así, los días y las noches se sucedían por voluntad divina, como era designio de Dios que los seres humanos caminaran y hablasen y tuvieran una marcada aversión hacia las serpientes, quienes, por supuesto, reptaban porque así lo había dictado el plan divino, al igual que había determinado que el sol diera luz y calor, que tras la lluvia apareciera el arco iris o que se sucedieran las estaciones. Pero con el paso del tiempo y con el progreso de lo que llamamos Ciencia, personalizado en figuras como Theodoric von Friberg (descubridor del fundamento del arco iris en el siglo XV), Copérnico, Galileo, Newton, Faraday, Halley, Darwin, Einstein y tantos otros, el conocimiento humano se ha ido expandiendo, haciendo que esos huecos sean cada vez más angostos o simplemente se rellenen de conocimiento. De esta manera los parones de la explicación científica son menos frecuentes e insuperables y por lo tanto, es menos necesaria la invocación a una voluntad de orden superior para dar cuenta algo que la Ciencia es incapaz de esclarecer.

Paréntesis: en la asistencia sanitaria, la psique, lo psicosomático o simplemente los nervios, vienen a ser el equivalente del Dios de los huecos de Tyson. Esto sucede a menudo, primero, al nivel particular del paciente con sintomas de expresión somática para los que por el motivo que sea no se encuentra un origen y que es por ello remitido a Salud Mental con la etiqueta de somatizador a pesar de que con el tiempo, la dedicación y el interés suficientes pudiera encontrársele una enfermedad fisica claramente relacionada con su malestar (1). Puede asi diagnosticarse de "histeria" a una mujer portadora de dos síntomas somáticos peculiares (boca ardiente y vulvodinia) para los que por su aparición en la misma persona y de una manera prácticamente simultánea también podría 
MÁRGENES DE LA PSIQUIATRÍA Y HUMANIDADES

considerarse como explicación alternativa un desequilibrio microbiano inducido por amoxicilina-clavulánico o alguna otra bomba antibacteriana. A un nivel más general, la "Psicosomática" ha sido un eficaz rellenahuecos en la explicación, entre otras, de la úlcera gástrica (considerada ahora una enfermedad infecciosa, lo que, por cierto, borra de un plumazo diferencias individuales), mientras que la atribución a factores psicológicos del origen de la fibromialgia -la enfermedad que gozó de menos consideración en una encuesta entre médicos y estudiantes de Medicina de un país serio como Noruega (2)- sirve para que las personas afectadas sufran no solo los sintomas, sino el hecho de ser muy frecuentemente etiquetadas de marineuras y que sean sistemáticamente derivadas a centros de Salud Mental, en una actitud que sería parangonable a la derivación a Medicina Interna o Infecciosas de toda persona diagnosticada de esquizofrenia, basándonos en una no demostrada teoría vírica de la enfermedad. En el caso de la fibromialgia, además, se trata de un padecimiento mayoritariamente femenino, con lo que para algunos se resuelve el enigma de dónde se ha escondido la "histeria" en nuestros tiempos. Y ya tenemos el hueco rellenado, sin necesidad de invocar a más dioses que a la tradición psiquiátrica (que, hay reconocerlo, es una divinidad poderosa).

Cerrado el paréntesis, el Dios de los huecos de Tyson revela, en el fondo, la necesidad humana de encontrar respuestas y explicaciones. El humán, como llama Jesús Mosterín al ser humano individual (3), observa, descubre, y busca relaciones entre sus observaciones. No solo percibe que al día le sigue la noche, sino que siempre ha intentado buscar una explicación para esta sucesión. O para la sucesión de eventos desfavorables que le ha golpeado durante un tiempo (¿será ese gato negro con el que me crucé?
¿La mirada torva de mi vecina? ¿Puede ser que olvidase santiguarme al salir de casa?).

Otro divulgador científico, Michael Shermer, habla de que la estructura cognitiva de nuestra especie nos obliga de alguna manera a encontrar lo que él llama "agenticity" y "patternicity" (4). La primera, que traduciremos libre y cacofónicamente como "agentidad", es la tendencia humana a creer que el mundo está controlado por agentes invisibles intencionales, es decir, dotados de una intención, de una disposición o voluntad a hacer que las cosas que vemos y vivimos sean de una determinada forma. La agentidad está en la base de la atribución a los dioses (o al Dios judeocristiano) de la estructura y el funcionamiento del cosmos, por lo que enlaza con el Dios de los huecos de Tyson, pero también sirve para atribuir determinados sucesos al diablo, a los espíritus, a las ánimas, a las meigas o a las sorgiñes, según de donde sea cada cual. El segundo elemento lo traduciremos por "búsqueda de estructura"; sí: es una traducción horrible, pero no me ocurre nada mejor, y suena menos mal que agentidad, ¿no? Para que no haga tanto daño a la vista al leerlo lo convertiré en BdE. La BdE es la tendencia a encontrar patrones o estructuras significativas en lo que en realidad es un ruido sensorial o perceptivo carente de significado. Esta tendencia cognitiva está claramente emparentada con la apofenia de Conrad, esa experiencia consistente en ver patrones, conexiones, o ambos, en sucesos aleatorios o datos sin sentido y que sirve para que creamos encontrar vinculaciones entre sucesos que sustentan, por ejemplo, creencias supersticiosas, como en los ejemplos del párrafo anterior. Evidentemente, la experiencia delirante, por lo mucho que tiene de encaje forzado de piezas, no deja de ser una expresión máxima de la $\mathrm{BdE}$, y la ideación de influencia, una variante patológica de la agentidad. 
MÁRGENES DE LA PSIQUIATRÍA Y HUMANIDADES

¿A qué viene este rollo? Pues a comentar que la Ciencia, el conocimiento humano, está siempre en construcción, y que los humanos no siempre nos acordamos de ello cuando tenemos que explicar nuestros hallazgos. En términos de Tyson, tal vez rellenemos los huecos precipitadamente, creyendo erróneamente que ya hemos dado con la respuesta al enigma de que se trate. Los humanes tenemos una tendencia más que sospechosa a considerar que lo que sabemos a cada momento es lo definitivo, lo cual es un craso error. En Medicina, incluso en la seria, objetivable y tangible (es decir, no ya en la menos concreta, más etérea y menos medible Psiquiatría), lo que hoy es cierto mañana puede no serlo. Recientemente se ha puesto en cuestión que la dieta grasa sea perjudicial para la salud (5) o que el ejercicio mejore la supervivencia en relación con el sedentarismo (6). Y un cercanísimo trabajo realizado en el Reino Unido (7) concluye que los pregonados efectos provechosos de la ingesta de cantidades moderadas de alcohol solo son aplicables a un grupo muy definido de humanes: el de las mujeres mayores de 65 años, lo que contraviene esa pieza de saber popular que dice que "ahora que estamos solos, ahora que nadie nos ve, beberemos un buen vino, igual que hacía Matusalén", ya que si bien es cierto que el humán a quien alude la canción tenía bastantes más que 65 años, a juzgar por las fuentes consultadas era varón. Este wealth of evidence que desmonta dogmas previos no termina de impresionar, sin embargo, a los jurásicos que por llevar unos cuantos años en esto ya hemos asistido a revelaciones como que -lo mencionábamos antes- de repente hay bacterias en el estómago, de un día para otro resulta que las neuronas se regeneran o de pronto tenemos que empezar a hacernos a la idea de que existen mecanismos inflamatorios allá donde antes no los imaginábamos (por ejemplo, en la depresión, y volveremos sobre ello). Siento repetirme, pero los hallazgos de Poynard y colaboradores (8) vienen al pelo a este respecto. Los autores seleccionaron artículos originales y metaanálisis publicados entre 1945 y 1999 acerca de las hepatitis y las cirrosis. Una vez reunido tan voluminoso cuerpo de saber, lo evaluaron a la luz de los conocimientos en este campo en 2000, y encontraron algo ciertamente demoledor: sólo el $60 \%$ de las conclusiones de los trabajos estudiados originales eran ciertas y el restante $40 \%$ se repartía casi a la par entre conclusiones obsoletas y meramente falsas, de modo que pudieron calcular que la vida media de las verdades en Medicina (en un campo "objetivable" como es la hepatología) no pasa de 45 años, y que al ritmo actual dentro de 50 años sólo pervivirá el $26 \%$ de los dogmas actuales. Uno tiene la impresión de que si intentásemos replicar estos hallazgos en Psiquiatría los resultados no serán mejores, ni mucho menos. Una vez más, hay que concluir que los valores que deberían presidir nuestra práctica son la prudencia y la humildad.

Pues bien: retomando a Tyson y Shermer, uno podría aventurar que de la misma manera que en el pasado se invocaba a Dios para rellenar la laguna de nuestros conocimientos, en nuestros días, con la consagración de la Ciencia como la llave para el conocimiento, se tiende a explicar cualquier hallazgo acudiendo a dogmas que no son sino verdades provisionales que no han superado la categoría de hipótesis. El dios de los huecos, no es el conocimiento científico, sino lo que creemos conocer de la Ciencia. Para ilustrar esto, nada mejor que el ejemplo del siempre cumplidor litio, por el que uno debe reconocer que siente debilidad. A este simple (en el mejor de los sentidos) elemento le ha descubierto una riada de efectos bio- 
MÁRGENES DE LA PSIQUIATRÍA Y HUMANIDADES

químicos y biológicos, si bien algunos de ellos solo acontecen a concentraciones tóxicas del producto. Como señala Belmaker (9), esa versatilidad ha permitido al litio alinearse con las teorías al uso a cada momento en materia de la fisiopatología y fisiología del trastorno bipolar. En la década de 196070 , en que las monoaminas eran el objeto de moda en investigación, se pudo demostrar que el litio incrementaba los metabolitos desaminados de la noradrenalina (10). En la de 1970-1980, en la que los receptores de neurotransmisores eran lo más de lo más, se pudo probar que el litio prevenía la supersensibilidad de los receptores dopaminérgicos (11). En torno a 1980-1990, la era de los segundos mensajeros, se descubrió la acción del litio tanto sobre la adenil ciclasa (12) como sobre la inositol monofosfatasa (13). En la recta final del siglo XX, en que se impuso la investigación sobre los terceros mensajeros, se observó que el litio afecta al c-fos y c-jun (14) e incrementa la unión del DNA a la proteína CREB DNA influyendo en la actividad de determinados genes ligados supuestamente al trastorno bipolar (15). Y llegado el siglo XXI, y en consonancia con lo que mola en la actualidad, se ha demostrado el efecto neuroprotector del litio a nivel neurocientífico (16) y clínico (17). Esta propiedad abre la posibilidad de que el litio pueda tener utilidad en el tratamiento de la enfermedad de Alzheimer (18). Además, el litio inhibe la glucógeno sintasa quinasa 3 (GSK-3, para los amigos), una enzima que se postula implicada en el trastorno bipolar y que también se supone que se dedica a tiempo parcial a enredar en relación con el péptido beta-amiloide y la proteína $\tau$ hiperfosforilada, a su vez implicados en la fisiopatología del Alzheimer (19). Además, la GSK-3 es una enzima proinflamatoria, por lo que -bingo- alguno de los efectos clínicos del litio podrían tener que ver con su acción indirectamente antiinflamatoria (20). Puesto que ahora mismo está en la cresta de la ola el interés por los mecanismos inflamatorios en los trastornos afectivos y en particular en la depresión, se confirma aunque no sabemos qué hace el litio, podemos estar seguros de que ningún otro fármaco es más capaz que él de complacer a los investigadores, ya que da sentido a la hipótesis en boga a cada momento. Quod erat demostrandum. Y, para asombro de quien sea capaz de asombrarse, esta desinteresada actuación en pro de la Ciencia y de los científicos la lleva a cabo el litio sin darse importancia, sin pasar de ser un minúsculo elemento con número atómico 3 al que no promocionan ni sus fabricantes. En reconocimiento a este detalle le voy a dedicar la primera tabla que incluyo en estos comentarios después de varios años dando la vara, hombre; es la Tabla 1, no tiene pérdida, seguro que el lector avezado o la lectora avezada la encuentra con facilidad. Por retomar a Tyson, las sucesivas explicaciones son una manera de rellenar la sorpresa por la efectividad del litio. Y si recordamos a Shermer, la manera de dar un sentido al patrón de respuesta al producto que se ha apreciado desde su introducción en la terapéutica psiquiátrica.

Un detalle interesante es que estas hipótesis tienen su traducción en eslóganes. Una vez que la Psicofarmacología ha alcanzado un cierto marchamo científico, ¿qué mejor forma de transmitir respetabilidad científica y evidencia de seriedad para un producto que ligarlo a una teoría en boga? Y en boga quiere decir lo que a cada momento convenga. Cualquiera que haya dispuesto de una suficiente perspectiva habrá comprobado que alguna empresa que vendió que detrás de la depresión había una disfunción serotoninérgica no tuvo problemas para años después remarcar la importancia de la noradrenalina 
MÁRGENES DE LA PSIQUIATRÍA Y HUMANIDADES

Tabla 1:

El complaciente litio (completada a partir de Belmaker)

\begin{tabular}{|l|l|l|}
\hline Época & Teoría en boga & Cumplimiento por Litio \\
\hline $1960-70$ & Monoaminas & Sí: Litio incrementa derivados desaminados de noradrenalina \\
\hline $1970-80$ & Receptores & Sí: litio previene la supersensibilidad de los receptores dopaminérgicos \\
\hline $1980-90$ & Segundos mensajeros & Sí: Acción sobre la adenil ciclasa y sobre la inositol monofosfatasa \\
\hline $1990-2000$ & Terceros mensajeros & $\begin{array}{l}\text { Sí: el litio afecta al c-fos y c-jun e incrementa la unión del DNA a la } \\
\text { proteína CREB DNA }\end{array}$ \\
\hline $2000-10$ & Neuroprotección & Sí: Efecto neuroprotector \\
\hline $2010 \ldots$ & Inflamación & Sí: Acción antiinflamatoria \\
\hline
\end{tabular}

a la hora de promocionar su antidepresivo "dual". La pena es que un producto que tenía en cartera como inhibidor de la recaptación de noradrenalina se ha quedado en el camino (21), porque de lo contrario, el foco de la explicación -de $s u$ explicación- de la depresión habría pasado a estar en la noradrenalina. Y a uno le da la impresión de que muchos prescriptores habrían aceptado sin la menor crítica ese viraje. El producto en cuestión, por cierto, se está empezando a estudiar como una alternativa en el TDAH (22), recuperando un camino ya recorrido por otra molécula del mismo propietario, que tras ser probado como un antidepresivo noradrenérgico en los 80 (23) pasó después a convertirse en uno de los medicamentos de referencia para esa indicación; en su viaje hacia una nueva indicación adquirió una "a" al comienzo del nombre con el que previamente era conocida.

La reconversión de moléculas de antidepresivos en medicamentos anti-déficit de atención no es reprochable en sí misma. Los primeros IMAOs no fueron sino tuberculostáticos a los que se apreció una acción timoléptica, como recordaremos, y la transformación a que dio lugar es mérito de los clínicos que apreciaron ese efecto. Lo que es llamativo es la invocación a teorías para sustentar el uso de los fármacos en determinadas indicaciones. Si el litio fuera un medicamento de interés comercial, que no lo es, para justificar su uso, cada cierto tiem- po las empresas interesadas en el producto expondrían a los prescriptores las sucesivas teorías que recoge la Tabla 1. La teoría, cuando se usa en la promoción de los fármacos, rellena (Tyson) la necesidad de dar base a la propuesta de usar el medicamento, o pretende dar sentido al patrón (Shermer) de efectividad o cuando menos de creciente uso de los medicamentos en Psiquiatría.

Pues bien, para redondear todas estas divagaciones, comentaremos tres artículos recientes que al suprascrito le parece que vienen al pelo para ilustrar estos asuntos; bueno, no se lo diga el lector o la lectora a nadie o a nadia, pero en realidad, todo el rollo previo viene a ser una forma para estirar lo que hubiera sido un comentario más breve de haberse reducido a los tres trabajos en cuestión. Las cosas, a veces, son así de tristes y de simples (aquí, lo de simple tiene un sentido menos honroso que el del litio).

Vamos allá: Caída en desgracia, por diversos motivos, la hipótesis dopaminérgica de la esquizofrenia, o la serotoninérgica de la depresión, ahora se dirige la mirada hacia las vías glutamatérgicas. El glutamato, como se sabe, es aminoácido y el neurotransmisor excitatorio por excelencia. También dio nombre a un grupo pop de los 80 , al que uno recuerda sobre todo por su versión en español (https://www.youtube. com/watch? $=0 \mathrm{zSl0lg} 92 \mathrm{Eo}$ ) del déserteur de Boris Vian (https://www.youtube.com/ 
MÁRGENES DE LA PSIQUIATRÍA Y HUMANIDADES

watch? $\mathrm{v}=$ gjndTXyk3mw\&list $=$ RDgjndTX yk3mw\#t=0). Y en su momento su sal monosódica fue ligada al llamado "síndrome del restaurante chino", un cuadro caracterizado por un cortejo sintomático que puede incluir migraña, rubefacción, sudor, y sensación de presión en la boca o cara. Por si el glutamato no reuniera ya pocos méritos, el síndrome del restaurante chino llegó a alcanzar honores de pregunta estúpidorebuscada de oposición al ser incluido en un examen MIR. Todo un carácter, pues, el glutamato, a pesar de que con el paso del tiempo se haya ido descartando que fuera el responsable del citado cuadro (24).

Hace algún tiempo comentábamos un estudio frente a placebo de Hallak y colaboradores (25) en el que la infusión intravenosa del arcaico antihipertensivo nitroprusiato se tradujo en una rápida (en materia de horas) y duradera (semanas) remisión de síntomas positivos y negativos de esquizofrenia. La rápida y sorprendente mejoría, que evoca la observada en depresiones graves con ketamina, no se ha podido relacionar con certeza con ningún mecanismo farmacodinámico, aunque los autores señalaban la posible implicación de los receptores NDMA y el sistema glutamatérgico. ¿Por qué? ¿Tal vez porque ahora el neurotransmisor que encontramos en todos los huecos es el glutamato? Por cierto, teniendo en cuenta que las sinapsis no dejan de ser huecos, gaps, en inglés, esta paridilla que se me ha ocurrido no deja de ser en ese idioma un pun, un juego de palabras. ¿O será porque en nuestra $\mathrm{BdE}$ encontramos glutamato por doquier? Chi lo sa...

La ketamina, a la que nombrábamos hace unas líneas, es un antagonista NMDA, lo que abre la posibilidad de que se desarrollen nuevos compuestos para la depresión que actúen de manera similar (26). También se ha considerado que la acetil cisteína po- dría ejercer sus variopintas acciones psicofarmacológicas a través de la modulación de vías glutamatérgicas (27). Sí, sin duda, el glutamato es actualmente el neurotransmisor de los huecos. En la esquizofrenia, en concreto, se trataría de potenciar la acción del receptor NMDA. Una forma hacerlo sería inhibir la D-amino ácido oxidasa (DAAO, en corto), un enzima con tan mala idea que se dedica a metabolizar la D-serina, que a su vez es un potente neurotransmisor coagonista en el receptor glutamatérgico NMDA.

Pues bien, otro producto con una posible acción glutamatérgica ensayado en la esquizofrenia es el benzoato de sodio. Esta sustancia, además de estar presente en la naturaleza en productos como la leche, es un quelante del amonio y como tal se emplea como medicamento en enzimopatías del ciclo de la urea (28). Pero también tiene un uso industrial como conservante, por sus propiedades antimicrobianas y antifúngicas. Como el benzoato sódico sólo es efectivo en condiciones ácidas, es un conservante alimentario adecuado sobre todo en conservas, en el vinagre, en bebidas carbonatadas (ricas en ácido carbónico), en productos ricos en ácido cítrico (mermeladas y zumos) y en salsas de comida china (soja, mostaza). Cada vez que el lector o lectora ingiere estos alimentos, que sepa que si son procesados es más que posible que se esté metiendo benzoato sódico para el cuerpo. Y como nadie es perfecto, ni siquiera los productos naturales quelantes del amonio que se emplean conservantes, el benzoato ha sido relacionado con diversos males, entre ellos, la posibilidad de que facilite la aparición de TDAH o esté relacionado con un incremento en la prevalencia del TDAH (si es que el aumento de los casos de este trastorno es un hecho epidemiológico y no una moda diagnóstica) (29-31). 
MÁRGENES DE LA PSIQUIATRÍA Y HUMANIDADES

No queda ahí la cosa: como muchas otras moléculas pluriempleadas, además de eliminar microbichos (el benzoato sódico, que todavía no lo habíamos contado, aparece en algunos populares antitusivos comercializados en España) este simpático conservante resulta ser un inhibidor de la DAOO, por lo que Lane y colaboradores decidieron estudiar si podría tener valor terapéutico añadirlo a la pauta antipsicótica habitual de un grupo de pacientes con esquizofrenia crónica (32). Según nos cuentan, y no tenemos por qué dudarlo, en comparación con quienes tomaron placebo, los pacientes que recibieron benzoato mostraron una mejoría del $21 \%$ en la PANSS y también tuvieron mejores resultados en sintomatología negativa, el GAF, en una escala de calidad de vida y en el CGI, por lo que los autores estiman que esta efectividad del benzoato muestra que merece la pena estudiar la posibilidad de desarrollar moléculas que se dediquen más específicamente a inhibir la DAOO.

Otro paréntesis: Como el lector sagaz y la lectora sagaza habrán notado, el benzoato, además de ejercer una sinergia glutamatérgica, está presente en salsas de uso frecuente en la comida china, por lo que igual resulta ser el verdadero causante del síndrome del restaurante chino. Que alguien estudie esto, que si sale que sí, tendremos un serio candidato a pregunta majadera de oposición.

Segundo artículo comentable: Nagele y colaboradores han demostrado que un clásiquísimo anestésico, el óxido nitroso $\left(\mathrm{N}_{2} \mathrm{O}\right)$, tiene un efecto terapéutico en la depresión refractaria al tratamiento (33). El procedimiento, en este caso, no es muy sport, ya que los pacientes inhalaron durante una hora $\mathrm{N}_{2} \mathrm{O}$ al $50 \%$ (los que recibieron placebo inhalaron nitrógeno al 50\%). Los pacientes fueron evaluados a las $2 \mathrm{y}$ a las
24 horas, y se observó una notable diferencia del efecto del $\mathrm{N}_{2} \mathrm{O}$ en comparación con el del placebo. Algunos efectos secundarios fueron un poco aparatosos (sobre 20 pacientes hubo un ataque de ansiedad, una regurgitación, dos cuadros de ansiedad, uno de claustrofobia), pero los autores nos tranquilizan argumentando que fueron de intensidad leva a moderada y que no se apreció un incremento en la homocisteína plasmática, lo que muestra que el $\mathrm{N}_{2} \mathrm{O}$ produjo una mínima o nula inactivación del metabolismo dependiente de vitamina $\mathrm{B}_{12}$. ¿Cómo funciona el $\mathrm{N}_{2} \mathrm{O}$ ? Pues como su analogía con el efecto antidepresivo de la ketamina podría sugerir, los autores nos recuerdan que se trata de un antagonista NMDA, lo que nos lleva de nuevo al glutamato, neurotransmisor de los huecos. Tiene su aquél, por cierto, que el clásicamente conocido como "gas de la risa" sea un antidepresivo de efecto ultrarrápido. El día que el dato llegue a la prensa será difícil desmontar esa creencia tan generalizada en la población de que para ayudar a los deprimidos es útil contarles chistes o hacerles gracietas. Es posible que con el fin de evitar el recurso al fármaco haya quien proponga ir directo al grano y hacer cosquillas a los pacientes para que se rían. Sería la cosquilloterapia; más técnico quedaría gargaletoterapia (de $\gamma \alpha \rho \gamma \alpha \lambda \eta \tau o ́$, hacer cosquillas; en griego todo parece de más fuste).

Eso que el que un antiséptico tenga una acción terapéutica en la esquizofrenia y el gas de la risa lo tenga en la depresión dejan ciertamente el listón muy alto. Pero el tercer artículo, mire Ud. por dónde, tiene un no-se-qué (o a lo peor, un sí-sé-qué) que lo hace inigualable. Es un estudio relacionado con el acamprosato, un fármaco de acción consolidante de la abstinencia de alcohol que parece parcialmente relacionada con el inevitable glutamato de los huecos y que resulta más eficaz en pacientes con determina- 
MÁRGENES DE LA PSIQUIATRÍA Y HUMANIDADES

dos marcadores de un gen que codifica una porción de -bingo, de nuevo- del receptor NMDA (34).

Paréntesis: Lo del glutamato como rellenahuecos y generador de patternicity tiene el sólido apoyo de que, al menos en cerebro de ratas, se ha comprobado que el litio ejerce una acción reguladora sobre el sistema glutamatérgico, a la cual se podría atribuir el efecto eutimizante del catión (35). De nuevo el litio presto a justificar la invocación a cualquier agente o teoría para rellenar nuestros huecos de conocimiento. Tendría que haberlo incluido en la Tabla 1.

Pues bien: Spanagel y colaboradores nos cuentan los resultados de un llamativo estudio en el que concluyen que el acamprosato no es la parte activa del acamprosato cálcico (36). Y si no lo es, solo cabe que lo sea el calcio, como el lector lince y la lectora linza habrán al punto deducido. El acamprosato es la sal cálcica del N-acetilhomotaurinato, un compuesto relacionado con la taurina. A Spanagel y colaboradores se les ocurrió ver cuál era el efecto de la sal sódica (es decir, del acamprosato sódico) en varios modelos animales, y comprobaron que no era en absoluto efectiva. Eso hacía pensar que el secreto de la acción terapéutica estaba en el calcio $\mathrm{y}$, a modo de verificación, ensayaron otra sal, el gluconato de calcio, en los mismos modelos, y observaron efectividad antialcohólica. Es más: trasladaron sus pesquisas a la clínica humana y encontraron que los pacientes con calcemias más elevadas derivadas del tratamiento con acamprosato cálcico mostraban una mejor evolución según parámetros tales como el tiempo transcurrido hasta recidiva del consumo o la abstinencia acumulada. Con estos datos solo podían concluir que el N-acetil-homotaurinato es una molécula biológicamente inactiva y que actúa como algo así como de vehículo del calcio. Lo cierto es que ya que hay al menos un simpático antecedente, el del ácido valproico, cuya actividad antiepiléptica se puso de relieve al utilizarlo para disolver otro compuesto del que se esperaba una acción anticonvulsivante. Puesto que el nuevo producto, que demostró efectividad efecto en dicha solución, no fue eficaz cuando era disuelto en otras sustancias, se dedujo que la molécula realmente anticomicial era el disolvente (el valproico) y no el supuestamente activo, lo que constituye un ejemplo más de los muchos hallazgos casuales o serendípicos que jalonan la historia de la Farmacología (37). Pero aun contando con este precedente, en el caso del acamprosato lo llamativo es que el descubrimiento se ha producido después de la comercialización, momento en que, como dicen los autores, sus efectos, descritos "en más de 450 publicaciones originales y ensayos clínicos y en un millón y medio de pacientes pueden tener que ser atribuidos al calcio" en lugar de a la supuesta molécula activa.

Y ahora, para cerrar, la moralina. El conocimiento humano ha avanzado mucho y sigue avanzando, y cada vez es menos necesario invocar a designios, planes o actuaciones (agentidad) divinos para explicar los huecos de ignorancia que encontramos en nuestro camino, básicamente, porque cada vez encontramos menos huecos. Pero eso no quiere decir que no encontremos huecos, ni que dejemos de actuar como humanes que necesitan rellenarlos o encontrar estructura (patternicity) en el cosmos para poder aprehenderlo y relacionarse con él. La Ciencia $\mathrm{y}$ sus hallazgos provisionales se han convertido en los actuales rellenahuecos, con modelos, hipótesis y teorías que pretenden dar respuestas definitivas cuando no sabemos si realmente existen tales respuestas. También se recurre a ellas para entrever un determinado patrón allí donde no es posi- 
MÁRGENES DE LA PSIQUIATRÍA Y HUMANIDADES

ble encontrarlo. Las supuestas respuestas y los posibles patrones, además, tienen vocación de ser definitivos, únicos, de no contemplar otras variables o posibilidades que ellos mismos. Habría que mantenerse alerta y no olvidar que el saber está siempre en construcción, y por ello tiene más sentido pensar que nos acercaremos cada vez más a cerrar los huecos que a terminar de taparlos definitivamente. Trasladado al ámbito de la relación clínica, no debe perderse de vista el conocimiento o la evidencia que sustentan nuestras intervenciones son siempre provisionales, por lo que en realidad más que de lo que sabemos habría que hablar de lo que a día de hoy creemos saber. Hay que recordar que lo que sostiene nuestra práctica es lo que más a mano tenemos para vadear nuestras dudas y nuestra ignorancia y la racionalidad de la Ciencia queda siempre matizada por nuestra estructura cognitiva necesitada de rellenar huecos y encontrar respuestas y patrones fáciles de entender. El obligado corolario es que por todo ello los valores que han de presidir la actividad asistencial son, de nuevo, la prudencia y la humildad.

Bibliografía

(1) Rougier SD. "C'est psychosomatique". Est-ce le bon diagnostic? Paris: Albin Michel, 2008

(2) Album D, Westin S. Do diseases have a prestige hierarchy? A survey among physicians and medical students. Soc Sci Med. 2008; 66: 1828 [Texto completo en: http://www. sciencedirect.com/science/article/pii/ S0277953607003929\#].

(3) Mosterín J. La naturaleza humana. Madrid: Espasa Calpe, 2008

(4) Shermer M. The Believing
Brain: From Ghosts and Gods to Politics and Conspiracies - How We Construct Beliefs and Reinforce Them as Truths. New York: Times Books, 2011

(5) Harcombe Z, Baker JS, Cooper SM, Davies B, Sculthorpe N, DiNicolantonio $\mathrm{JJ}$, et al. Evidence from randomised controlled trials did not support the introduction of dietary fat guidelines in 1977 and 1983: a systematic review and meta-analysis. Open Heart 2015; 2(1): e000196 [Texto completo en: http://www.ncbi.nlm.nih.gov/pmc/ articles/PMC4316589/]

(6) Biswas A, Oh PI, Faulkner GE, Bajaj RR, Silver MA, Mitchell MS, et al. Sedentary Time and Its Association With Risk for Disease Incidence, Mortality, and Hospitalization in Adults: A Systematic Review and Meta-analysis. Ann Intern Med 2015; 162: 123-32 [Abstract en: http://www. ncbi.nlm.nih.gov/pubmed/25599350].

(7) Knott CS, Coombs N, Stamatakis E, Biddulph JP. All cause mortality and the case for age specific alcohol consumption guidelines: pooled analyses of up to 10 population based cohorts. BMJ 2015 Feb 10;350:h384. doi: 10.1136/bmj.h384 [Texto completo en: http://www.bmj.com/ content/350/bmj.h384].

(8) Poynard T, Munteanu M, Ratziu V, Benhamou Y, Di Martino V, Taieb J et al. Truth survival in clinical research: an evidence-based requiem? Ann Intern Med 2002;136: 888-895 [Texto completo en: http:// www.planetadoctor.com/documentos/MBEherramienta/13.pdf]

(9) Belmaker RH. Bipolar disorder. N Engl J Med 2004; 351: 476-86.

(10) Knapp S, Mandell AJ. Short- and longterm lithium administration: effects on the brain's serotonergic biosynthetic systems. Science 1973; 180: 645-7 [Abstract en: http:// www.ncbi.nlm.nih.gov/pubmed/4700609].

(11) Pert A, Rosenblatt JE, Sivit C, 
MÁRGENES DE LA PSIQUIATRÍA Y HUMANIDADES

Pert CB, Bunney WE Jr. Long-term treatment with lithium prevents the development of dopamine receptor supersensitivity. Science 1978; 201: 171-3 [Abstract en: http://www. ncbi.nlm.nih.gov/pubmed/566468].

(12) Ebstein R, Belmaker R, Grunhaus L, Rimon R. Lithium inhibition of adrenalinstimulated adenylate cyclase in humans. Nature 1976; 259: 411-3.

(13) Berridge MJ, Downes CP, Hanley MR. Lithium amplifies agonist-dependent phosphatidylinositol responses in brain and salivary glands. Biochem J 1982; 206:5 8795 [Texto completo en: http://www.biochemj. org/bj/206/0587/2060587.pdf].

(14) Leslie RA, Moorman JM. Potentiation of immediate-early gene c-fos expression in cerebral cortex by chronic lithium treatment after 5-HT2A receptor activation. En: Manji HK, Bowden CL, Belmaker R, eds. Bipolar medications: mechanisms of action. Washington, D.C.: American Psychiatric Press, 2000: 249-70.

(15) Unlap MT, Jope RS. Lithium attenuates nerve growth factor-induced activation of AP-1 DNA binding activity in PC12 cells. Neuropsychopharmacology 1997; 17: 12-7 [Abstract en: http://www.ncbi.nlm. nih.gov/pubmed/9194045].

(16) Manji HK, Moore GJ, Chen G. Lithium at 50: have the neuroprotective effects of this unique catión been overlooked? Biol Psychiatry 1999; 46: 929-40 [Abstract en: http:// www.ncbi.nlm.nih.gov/pubmed/10509176].

(17) Diniz BS, Machado-Vieira R, Forlenza OV. Lithium and neuroprotection: translational evidence and implications for the treatment of neuropsychiatric disorders. Neuropsychiatr Dis Treat. 2013; 9: 493-500 [Texto completo en: http://www. dovepress.com/lithium-and-neuroprotectiontranslational-evidence-and-implications-fopeer-reviewed-article-NDT].

(18) Forlenza OV, de Paula VJ,
Machado-Vieira R,Diniz BS, Gattaz WF. Does lithium prevent Alzheimer's disease? Drugs Aging 2012; 29: 335-42 [Abstract: http:// www.ncbi.nlm.nih.gov/pubmed/22500970].

(19) Shulman KI. Lithium for older adults with bipolar disorder: Should it still be considered a first-line agent? Drugs Aging 2010; 27: 607-15 [Abstract en: http://www. ncbi.nlm.nih.gov/pubmed/20658789].

(20) Beurel E, Jope RS. Inflammation and lithium: clues to mechanisms contributing to suicide-linked traits. Transl Psychiatry 2014; 4: e488 [Texto complete en http:// www.nature.com/tp/journal/v4/n12/full/ tp2014129a.html]

(21) Ball S, Dellva MA, D'Souza DN, Marangell LB, Russell JM, Goldberger C. A double-blind, placebo-controlled study of edivoxetine as an adjunctive treatment for patients with major depressive disorder who are partial responders to selective serotonin reuptake inhibitor treatment. J Affect Disord 2014; 167: 215-23 [Abnstract en: http://www. ncbi.nlm.nih.gov/pubmed/24995890].

(22) Markowitz JS, Brinda BJ. A pharmacokinetic evaluation of oral edivoxetine hydrochloride for the treatment of attention deficit-hyperactivity disorder. Expert Opin Drug Metab Toxicol 2014; 10: 128999 [Abstract: http://www.ncbi.nlm.nih.gov/ pubmed/25019201].

(23) Zerbe RL, Rowe H, Enas GG, Wong D, Farid N, Lemberger L. Clinical pharmacology of tomoxetine, a potential antidepressant. J Pharmacol Exp Ther 1985; 232: 139-43 [Abstract: http://www.ncbi.nlm. nih.gov/pubmed/3965689].

(24) Geha RS, Beiser A, Ren C, Patterson R, Greenberger PA, Grammer $\mathrm{LC}$, et al. Review of alleged reaction to monosodium glutamate and outcome of a multicenter double-blind placebo-controlled study. J Nutr 2000; 130(4S Suppl): 1058S-62S [Texto completo en: http://jn.nutrition.org/ 
MÁRGENES DE LA PSIQUIATRÍA Y HUMANIDADES

content/130/4/1058.long].

(25) Hallak JE, Maia-de-Oliveira JP, Abrao J, Evora PR, Zuardi AW, Crippa JA, et al. Rapid improvement of acute schizophrenia symptoms after intravenous sodium nitroprusside: a randomized, doubleblind, placebo-controlled trial. JAMA Psychiatry 2013; 70: 668-76. [Texto completo en: http://archpsyc.jamanetwork.com/article. aspx?articleid=1686035].

(26) Dutta A, McKie S, Deakin JF. Ketamine and other potential glutamate antidepressants. Psychiatry Res 2014. pii: S0165-1781(14)00861-0 [Abstract en: http:// www.ncbi.nlm.nih.gov/pubmed/25467702]

(27) Dean O, Giorlando F, Berk M. N-acetylcysteine in psychiatry: current therapeutic evidence and potential mechanisms of action. J Psychiatry Neurosci 2011; 36:7886 [Texto completo en: http://www.ncbi.nlm. nih.gov/pmc/articles/PMC3044191/].

(28) Degirmencioglu H, Oncel MY, Yurttutan S, Ekmen S, Suna Oguz S, Uras $\mathrm{N}$, Dilmen U. Citrulinemia de presentación atípica: hipo persistente. A propósito de un caso. Arch Argent Pediatr 2014; 112: e2068 [Texto completo en: http://www.sap.org. ar/docs/publicaciones/archivosarg/2014/ v112n5a15.pdf].

(29) Bateman B, Warner JO, Hutchinson E, Dean T, Rowlandson P, Gant C, et al. The effects of a double blind, placebo controlled, artificial food colourings and benzoate preservative challenge on hyperactivity in a general population sample of preschool children. Arch Dis Child 2004; 89: 506-11 [Texto completo en: http:// adc.bmj.com/content/89/6/506.long].

(30) McCann D, Barrett A, Cooper A, Crumpler D, Dalen L, Grimshaw K, et al. Food additives and hyperactive behaviour in 3 -year-old and 8/9-year-old children in the community: a randomised, double-blinded, placebo-controlled trial. Lancet 2007; 370:
1560-7 [Abstract en: http://www.ncbi.nlm. nih.gov/pubmed/178254050].

(31) Beezhold BL, Johnston CS, Nochta KA. Sodium benzoate-rich beverage consumption is associated with increased reporting of ADHD symptoms in college students: a pilot investigation. J Atten Disord 2014; 18: 236-41 [Abstract en: http://www. ncbi.nlm.nih.gov/pubmed/22538314]

(32) Lane HY, Lin $\mathrm{CH}$, Green MF, Hellemann G, Huang CC, Chen PW, et al.Addon treatment of benzoate for schizophrenia: a randomized, double-blind, placebo-controlled trial of D-amino acid oxidase inhibitor. JAMA Psychiatry 2013; 70: 1267-75 [Texto completo en: http://archpsyc.jamanetwork.com/article. aspx?articleid=1746121].

(33) Nagele P, Duma A, Kopec M, Gebara MA, Parsoei A, Walker M, et al. Nitrous Oxide for Treatment-Resistant Major Depression: A Proof-of-Concept Trial. Biol Psychiatry. 2014 Dec 9. pii: S0006-3223(14)00910-X. doi: 10.1016/j. biopsych.2014.11.016 [Texto completo en: http://www.biologicalpsychiatryjournal.com/ article/S0006-3223(14)00910-X/pdf].

(34) Karpyak VM, Biernacka JM, Geske JR, Jenkins GD, Cunningham JM, Rüegg $\mathrm{J}$, et al. Genetic markers associated with abstinence length in alcohol-dependent subjects treated with acamprosate. Transl Psychiatry 2014; 4: e462 [Texto completo en: http://www.nature.com/tp/journal/v4/n10/ full/tp2014103a.html].

(35) Dixon JF, Hokin LE. Lithium acutely inhibits and chronically up-regulates and stabilizes glutamate uptake by presynaptic nerve endings in mouse cerebral cortex. Proc Natl Acad Sci U S A 1998; 95: 8363-8 [Texto completo en: http://www.pnas.org/ content/95/14/8363.full].

(36) Spanagel $\mathrm{R}$, Vengeliene $\mathrm{V}$, Jandeleit B, Fischer WN, Grindstaff K, Zhang $\mathrm{X}$, et al. Acamprosate produces 
its anti-relapse effects via calcium. in a new World - 1952-1968". En Healy D Neuropsychopharmacology 2014; 39: 783-91 (ed): Thepsychopharmacologists Vol 3, [Texto completo en: http://www.nature.com/ London: Arnold, 2000; p 1-54.] npp/journal/v39/n4/full/npp2013264a.html].

(37) Comité Lyonnais de RecherchesThérapeutiques en Psychiatrie. The birth ofpsychopharmacotherapy: explorations 